\title{
Born with an Ear for Dialects? Structural Plasticity in the Expert Phonetician Brain
}

\author{
Narly Golestani, ${ }^{1,2}$ Cathy J. Price, ${ }^{3}$ and Sophie K. Scott ${ }^{1}$ \\ ${ }^{1}$ Institute of Cognitive Neuroscience, University College London, WC1N 3AR London, United Kingdom, ${ }^{2}$ Department of Fundamental Neuroscience, \\ University Medical School, University of Geneva, CH-1211 Geneva, Switzerland, and ${ }^{3}$ Wellcome Trust Centre for Neuroimaging, WC1N 3BG London, \\ United Kingdom
}

Are experts born with particular predispositions, or are they made through experience? We examined brain structure in expert phoneticians, individuals who are highly trained to analyze and transcribe speech. We found a positive correlation between the size of left pars opercularis and years of phonetic transcription training experience, illustrating how learning may affect brain structure. Phoneticians were also more likely to have multiple or split left transverse gyri in the auditory cortex than nonexpert controls, and the amount of phonetic transcription training did not predict auditory cortex morphology. The transverse gyri are thought to be established in utero; our results thus suggest that this gross morphological difference may have existed before the onset of phonetic training, and that its presence confers an advantage of sufficient magnitude to affect career choices. These results suggest complementary influences of domain-specific predispositions and experience-dependent brain malleability, influences that likely interact in determining not only how experience shapes the human brain but also why some individuals become engaged by certain fields of expertise.

\section{Introduction}

Expertise has been shown to have both functional and structural correlates in the human brain. For example, expert golfers show a different pattern of neural activity than novice golfers when planning shots (Milton et al., 2007), and London taxi drivers have a larger posterior hippocampal volume than matched controls (Maguire et al., 2000). It can be difficult to establish, however, the extent to which these effects relate to preexisting differences between the novice and expert groups, or whether these effects mainly arise from training-induced plasticity. Here we investigate brain anatomy in expert phoneticians_-individuals who are specialized in the study of phonetics. We aimed to distinguish experience-dependent plasticity from brain structural features that existed before the onset of expertise training.

Phoneticians typically spend one to four years of formal training learning to identify speech sounds and to transcribe them into an international phonetic alphabet. In many languages, there is no oneto-one correspondence between pronunciation and orthography, and narrow phonetic transcription involves parsing and identifying speech sounds so as to provide a direct correspondence between sound and symbol. Phoneticians are thus very quick and skilled at identifying even subtle regional accents. Unlike other kinds of expertise, such as musical skills, phonetic transcription skills are acquired

Received July 22, 2010; revised Dec. 14, 2010; accepted Jan. 7, 2011.

This work was supported by a Marie Curie International Incoming Fellowship from the European Commission awarded to N.G., and by Wellcome Trust grants to C.J.P. and S.K.S. We thank M. Eckert, C. Leonard, R. Amsel, R. Davis, B. Draganski, S. Kloppel, A. Brennan, J. Glensman, D. Bradbury, and the phoneticians who participated in this study for their help.

Correspondence should be addressed to Narly Golestani, University Medical School, 1 Rue Michel-Servet, CH1211 Geneva, Switzerland. E-mail: n.golestani@ucl.ac.uk.

DOI:10.1523/JNEUROSCI.3891-10.2011

Copyright $\odot 2011$ the authors $\quad 0270-6474 / 11 / 314213-08 \$ 15.00 / 0$ in adulthood, and we can precisely measure the amount of phonetic transcription training and experience. This allowed us to specifically test the effects on brain structure of extensive and naturalistic training (i.e., not in the laboratory) during adulthood.

To more generally characterize brain structural features underlying this form of language expertise, we also tested for brain structural differences between phoneticians and nonexpert controls. Functional brain imaging and transcranial magnetic stimulation studies have shown that the posterior portion of the left inferior frontal gyrus (the left pars opercularis) and the left supramarginal gyrus are involved in and even necessary for phonological processing and for subvocal rehearsal in verbal working memory (Paulesu et al., 1993; Zatorre et al., 1996; Burton et al., 2000; Nixon et al., 2004; Gough et al., 2005; Hartwigsen et al., 2010). Further, previous findings have shown that brain structure in the left auditory cortex, the parietal cortices, and the left inferior frontal cortex partly predicts individual differences in the perception and production of foreign speech sounds in healthy nonexperts (Golestani et al., 2002, 2007; Golestani and Pallier, 2007). We thus aimed to extend these normative findings to expertise by testing for group differences in left auditory, frontal, and parietal cortex morphology between phoneticians and nonphoneticians. Findings are discussed in terms of experience-dependent plasticity and of genetic studies suggesting differential degrees of experience-dependent malleability of different brain regions.

\section{Materials and Methods}

\section{Participants}

Seventeen phoneticians (11 women) and 16 age- and gender-matched controls (nine women) participated in the study. All participants were healthy (screened for neurological and psychiatric problems) and righthanded. The mean age of the phoneticians was 39.76 (SD, 13.17) years and that of the controls was 36.35 (SD, 10.55) years. Ten of the 17 pho- 
Table 1. Overview of morphometric analyses

\begin{tabular}{|c|c|c|c|c|}
\hline Analysis & Region & Measure(s) & Motivation & Results \\
\hline $\begin{array}{l}\text { Voxel-based morphometry: comparison } \\
\text { of gray matter and white matter } \\
\text { tissue-classified maps of phoneti- } \\
\text { cians and nonphoneticians }\end{array}$ & Whole brain & $\begin{array}{l}\text { Probability of gray and } \\
\text { of white matter }\end{array}$ & Exploratory first step & $\begin{array}{l}\text { Greater probability of white matter in right } \mathrm{HG}(t=3.34 \text {; } \\
P_{\text {FWE-co }}=0.05, \text { small-volume correction) and just posterior } \\
\text { to left HG ( } t=2.80 ; p<0.005 \text { uncorrected) in } \\
\text { phoneticians compared to controls (Fig. } 1)\end{array}$ \\
\hline $\begin{array}{l}\text { Macroanatomical labeling } \\
\text { a. Group difference } \\
\text { b. Correlations with years of phonetic } \\
\text { transcription training within phone- } \\
\text { ticians group }\end{array}$ & $\begin{array}{l}\text { Left and right } \\
\text { transverse gyri }\end{array}$ & $\begin{array}{l}\text { Volume } \\
\text { Gross morphology }\end{array}$ & $\begin{array}{l}\text { To elucidate above VBM results } \\
\text { Previous studies showing larger left } \\
\text { Heschl's gyrus and greater left } \\
\text { transverse gyrus splitting/dupli- } \\
\text { cation in faster compared to } \\
\text { slower phonetic learners }\end{array}$ & $\begin{array}{l}\text { a. Larger left }\left(t_{(31)}=2.02, p<0.05\right) \text { and right }\left(t_{(31)}=3.07 \text {, }\right. \\
\quad p<0.005) \text { transverse gyri in phoneticians compared to } \\
\text { controls (Figs. } 2,3 \text { ) } \\
\text { Phoneticians are more likely to have multiple or split left } \\
\quad\left(t_{(31)}=2.39, p<0.01\right) \text { but not right }\left(t_{(31)}=0.19, p>\right. \\
0.05) \text { transverse gyri than controls (Fig. } 4) \\
\text { b. No relationship between phonetic training and left or right } \\
\text { transverse gyrus volumes or degree of transverse gyrus } \\
\text { splitting within the phoneticians group }\end{array}$ \\
\hline Automatic measure of local gyrification & $\begin{array}{l}\text { Left and right } \\
\text { transverse gyri }\end{array}$ & Mean curvature & $\begin{array}{l}\text { To further confirm above gross } \\
\text { morphology results }\end{array}$ & $\begin{array}{l}\text { Trend towards less right }>\text { left asymmetry in local gyrification } \\
\text { the phoneticians compared to controls }\left(t_{(31)}=1.49,\right. \\
p=0.07)\end{array}$ \\
\hline $\begin{array}{l}\text { Automatic segmentation and labeling: } \\
\text { a. Group difference } \\
\text { b. Correlations with years of phonetic } \\
\text { transcription training within phone- } \\
\text { ticians group }\end{array}$ & $\begin{array}{l}\text { Left pars } \\
\text { opercularis } \\
\text { Left supramar- } \\
\text { ginal gyrus }\end{array}$ & $\begin{array}{l}\text { Gray matter volume } \\
\text { Surface area }\end{array}$ & $\begin{array}{l}\text { Known involvement in phonologi- } \\
\text { cal processing } \\
\text { Previous findings that structure left } \\
\text { prefrontal and inferior parietal } \\
\text { cortices in part predict phonetic } \\
\text { processing in healthy controls }\end{array}$ & $\begin{array}{l}\text { a. Larger left pars opercularis volume in phoneticians } \\
\text { compared to non-experts }\left(t_{(31)}=2.02, p<0.05 \text {, Bonfer- }\right. \\
\text { roni correction) } \\
\text { b. Years of transcription training predicts the surface area of } \\
\text { the left pars opercularis ( } r=0.64, p<0.01 \text {, Bonferroni } \\
\text { correction) } \\
\text { Similar trend for volume of the left pars opercularis ( } r=0.49 \text {, } \\
p=0.03 \text { ) (Fig. } 5 \text { ) }\end{array}$ \\
\hline
\end{tabular}

FWE-co, Family-wise error corrected.

neticians were native English speakers, four were early bilinguals (i.e., they started learning two languages in parallel early in life), and the remaining three were native speakers of a language other than English. All participants gave informed consent and the study was approved by the National Hospital and Institute of Neurology's joint medical ethics committee.

Phoneticians were recruited via a posting to a mailing list dedicated to phonetics. They worked in fields including academia, speech and language therapy, radio broadcasting (e.g., pronunciation experts), and forensics. Within the group, phoneticians had $1-9$ years (mean, $2.8 \pm 2.3$ years) of formal training in phonetic transcription. This range provided the necessary variation for a correlation that explicitly tested how brain structure was related to the number of years of phonetic training. The phoneticians' ability to learn unfamiliar phonetic contrasts was tested using a synthetic Hindi dental-retroflex contrast. Prior work has already established that the speed at which healthy (nonexpert) individuals learn new phonetic contrasts on this task is positively related to brain structure in left Heschl's gyrus (Golestani et al., 2007). The choice of this task allowed us to integrate the current study with previous findings. Details of the stimuli and training procedure can be found in Golestani et al. $(2002,2007)$. See Other findings, below for further details on the language backgrounds and early language experience of our participants.

\section{Image acquisition and analysis}

We used a 1.5 tesla Siemens Sonata MRI scanner to acquire high resolution, three-dimensional, T1-weighted images using a phased array coil (scanning parameters: matrix, $256 \times 224 \times 176$; isotropic resolution, 1 $\mathrm{mm}$; repetition time, $20.66 \mathrm{~ms}$; echo time, $8.42 \mathrm{~ms}$; inversion time, 640 $\mathrm{ms} ; \alpha=25^{\circ}$ ).

Table 1 provides an overview of the different analysis methods used, the motivation for using these, the regions and measures tested, and the results obtained.

Exploratory, whole-brain analysis: voxel-based morphometry Voxel-based morphometry (VBM) (Ashburner and Friston, 2000), implemented in SPM5 (Wellcome Department of Imaging Neuroscience, London, UK), was used to look for brain structural differences between phoneticians and controls. This method allows an exploratory search throughout the brain; it does not require subjective identification of anatomic boundaries and, hence, the use of arbitrary or conventional definitions of particular brain structures (Golestani et al., 2002).

For VBM, the anatomical images were processed in three steps, as follows: tissue segmentation, linear spatial normalization, and smoothing at $6 \mathrm{~mm}$. Note that VBM does not provide a direct measure of the volume or surface area of specific structures. Specifically, since VBM is based on tissue-segmented gray or white matter maps, it is particularly sensitive to differences in morphology that exist at gray/white matter boundaries, and such sensitivity may hide the presence of morphological differences that lie within a gray or white matter region or structure per se. Given that we complemented VBM with morphometric analyses, including manual and automatic volume and surface area extraction (see Image acquisition and analysis, below), we did not modulate the images (i.e., adjust for volume) during preprocessing. We compared the smoothed gray and white matter images between the phoneticians and controls on a voxel-by-voxel basis using independent samples $t$ tests. We used an uncorrected voxelwise threshold of $p<0.005$ with small-volume corrections in regions of interest predicted by previous studies, these being the left and right transverse gyri (Golestani et al., 2007). For the small volume corrections, we used the $40 \%$ thresholded probability maps of the macroanatomically defined left and right transverse gyri in the phoneticians.

\section{Hypothesis-driven, region of interest analyses}

Transverse gyri of the auditory cortex: macroanatomical labeling. We performed macroanatomical labeling on the left and right transverse gyri of the auditory cortex on anatomically normalized images using Anatomist (http://brainvisa.info/). This software allows simultaneous real-time viewing and voxel labeling on sagittal, coronal, and horizontal planes, and computation of three-dimensional volumes. We labeled multiple transverse gyri in cases where they existed. The choice of this labeling criterion was motivated by previous results showing gross morphology differences in this region between faster and slower phonetic learners (Golestani et al., 2007), and by the location of the VBM result in the left auditory cortex (see Results, below). Our transverse gyrus measure includes the subregion of the planum temporale that is adjacent to Heschl's gyrus. It is known that the primary auditory cortex (PAC) is usually confined to the transverse gyrus in cases where only one is present, but that when multiple transverse gyri are present, the more anterior one is most likely to include PAC but the most posterior one(s) is (are) likely to 


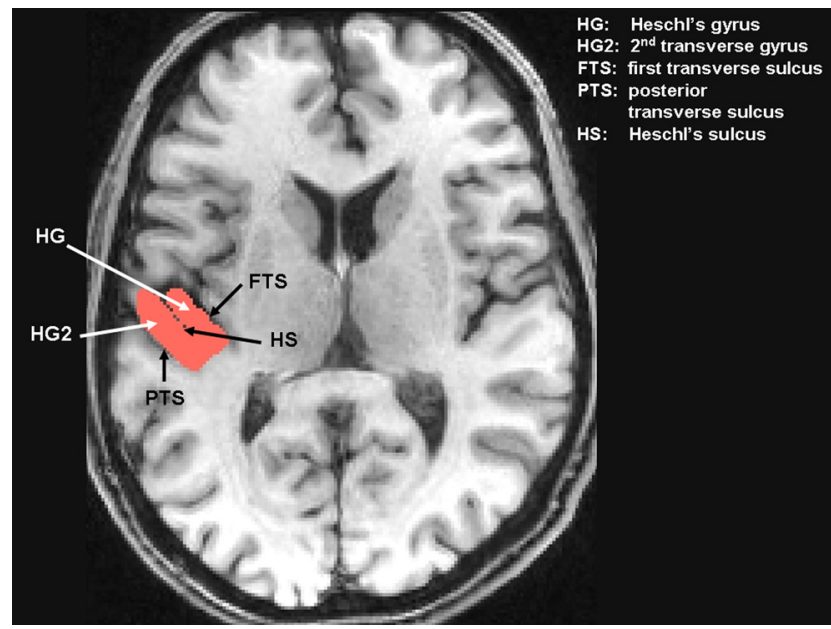

Figure 1. Transverse gyrus landmarks and boundaries shown on left hemisphere with two transverse gyri.

include secondary auditory cortex (Pfeifer, 1920; Rademacher et al., 2001). Hence, the planum temporale includes secondary (and tertiary, etc.) transverse gyri and, as such, our inclusion of multiple transverse gyri allowed us to simultaneously examine regions likely to include both the primary auditory cortex as well as planum temporale subregions directly adjacent to core primary auditory cortex.

The macroanatomical definition of the transverse gyrus used for labeling was as follows (Fig. 1). The first transverse sulcus (FTS) always constituted the anterior boundary. The posterior boundary was the most posterior transverse sulcus, this being posterior to second or third transverse gyri when they existed, or being Heschl's sulcus in the case of a single or split transverse gyrus. The medial boundary was drawn from the medial end of the FTS to the medial end of the most posterior transverse sulcus, and the inferior boundary was drawn along a plane running from the depth of the FTS to the depth of the posterior temporal sulcus or of Heschl's sulcus.

The rater was blind to participant group and to hemisphere, and the labeling was performed twice. The total (left and right) transverse gyrus volumes were significantly correlated across labeling sessions (Pearson's $r=$ 0.984 ), providing evidence for labeling reliability.

Transverse gyri of the auditory cortex: gross morphology. We quantified the gross morphology of the transverse gyri as follows. We gave a score of 1 if there was one transverse gyrus as viewed on a horizontal section, 2 if there were two, and 3 if there were three. We gave a score of 1.25 if the gyrus was split such that the intermediate sulcus (SI) extended to approximately one-quarter of the length of Heschl's gyrus (HG), 1.5 if it extended to approximately half the length of $\mathrm{HG}$, and 1.75 if it extended to approximately three-quarters the length of HG. Again, the rater was blind to group and to hemisphere, and the ratings were performed twice and were found to be reliable (Person's $r=0.948$ for the mean left vs right transverse gyrus splitting measure). Validation of the findings was further provided by the automated gyrification measure (see below).

Independent samples $t$ tests were used to compare transverse gyrus volumes and the degree of splitting between groups. Based on previous findings showing that faster phonetic learners have a larger left Heschl's gyrus and greater left transverse gyrus splitting or duplication than slower learners (Golestani et al., 2007), these tests were unidirectional. A measure of asymmetry [lateralization index (LI)] was then calculated on the manual gyrification measure $\left(G_{m}\right)$ for each participant as follows: $\mathrm{LI}=\left[\left(\mathrm{G}_{\mathrm{mR}}-\mathrm{G}_{\mathrm{mL}}\right) /\left(\mathrm{G}_{\mathrm{mR}}+\mathrm{G}_{\mathrm{mL}}\right) / 2\right]$, where $\mathrm{R}$ and $\mathrm{L}$ indicate right and left, respectively (cf. Penhune et al., 1996). Rightward asymmetries result in positive values, and local gyrification was considered to be asymmetric when $|\mathrm{LI}|>0.10$.

We also tested for experience-dependent differences in these measures within the phoneticians group. For this, we performed Pearson's correlations between years of formal phonetic transcription training (see Participants, above) and these measures.
Transverse gyri of the auditory cortex: automated measure of transverse gyrus local gyrification. To further examine gross morphology differences between groups, Freesurfer was used to automatically provide a measure of mean local gyrification (mean curvature) in the transverse gyri of all participants and in both hemispheres. LI was then calculated on the automated gyrification measure $\left(G_{a}\right)$ for each participant as follows: $L I=$ $\left[\left(G_{a R}-G_{a L}\right) /\left(G_{a R}+G_{a L}\right) / 2\right]$ (cf. Penhune et al., 1996). Rightward asymmetries result in positive values, and local gyrification was considered to be asymmetric when $|\mathrm{LI}|>0.10$.

\section{Frontal and parietal regions of interest}

Freesurfer (Fischl et al., 2004; Desikan et al., 2006) was used to automatically extract gray matter volumes and surface areas of the left pars opercularis and of the left supramarginal gyrus. We tested for differences between the phoneticians and controls in these measures using independent samples $t$ tests, and performed Bonferroni corrections for the number of regions of interest (ROIs) tested. Based on previous findings that a greater tissue density positively predicts individual differences in phonetic processing in these regions (Golestani and Pallier, 2007; Golestani et al., 2007), all tests were unidirectional. We also tested for experiencedependent differences in these measures within the phoneticians group. For this, we used partial correlations controlling for overall cortical volume to correlate years of phonetic transcription training with these measures. Given that partial correlations are sensitive to outliers, we excluded two outliers who had the most years of phonetic transcription training $(>2$ SDs above the mean) and who strengthened some of the results (see below).

We were not able to provide measures of surface area for the transverse gyri that are methodologically comparable to those derived for the supramarginal gyrus/pars opercularis because the Freesurfer software does not allow computation of surface area on regions that are imported from other sources, such as those defined by the macroanatomical labeling.

\section{Other findings}

\section{Language experience}

To statistically account for language experience in the phoneticians group, we evaluated language experience in the following manner. Each individual answered a detailed, nonstandardized questionnaire about their language experience background, including the number of languages that they had learnt formally and informally, the fluency with which they spoke each language, and the age at which they had acquired each language. The phoneticians were more multilingual than were controls. On average, they had had formal language instruction in up to 10 languages (mean number of languages, $5.6 \pm 2.1$ ), whereas the controls had had formal language instruction in up to four languages. Phoneticians also received more years of education than the controls (means: phoneticians, $19.29 \pm 1.64$ years in school plus university; controls, $15.23 \pm 3.56$ years; $t_{(31)}=3.85, p=0.001, n=33$, two-tailed). Knowledge of each language was weighted according to the age at which it had been learnt (higher weight for languages learnt earlier in life) and according to the fluency with which it was spoken (higher weight for more fluently spoken languages). The following weights were used: 1) proficiency: not fluent $=1$, somewhat fluent $=2$, moderately fluent $=3$, quite fluent $=4$, very fluent $=5$, native $=6 ; 2$ ) age of acquisition: ages $21+=$ 1 , ages $13-20=2$, ages $7-12=3$, ages $1-6=4$, at birth $=5$. A continuous language score was then calculated for each individual and this measure was used to determine whether language experience could account for some of the observed results.

\section{Educational level}

To ensure that the educational level of the phoneticians does not explain the structural effects (phoneticians had an average of $19.3 \pm 1.6$ years of education in total), we tested to see whether the number of years of education predicts various structural measures within the phoneticians.

\section{Relationship between auditory, frontal, and parietal cortex ROI} measures and years of transcription training, including two outliers

We repeated the partial correlations examining the relationship between various ROI measures and the amount of transcription training, including the two outliers (all 17 phoneticians). 


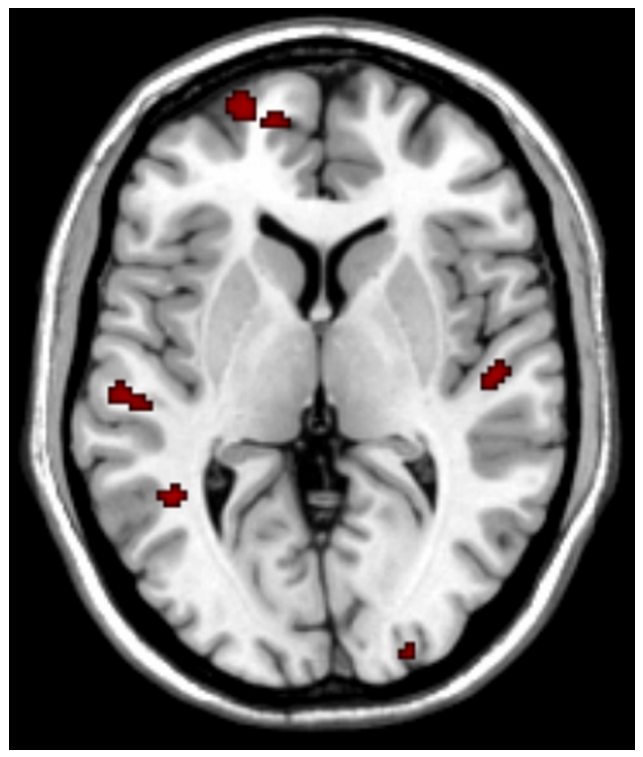

Figure 2. VBM anatomical difference in left and right transverse gyri: statistical parametric map showing greater white matter density in phoneticians compared with controls (MNI coordinates: left: $x=-56, y=-22, z=4$; and right: $x=50, y=-16, z=2$ ). Note that for display purposes, images are presented at a threshold of 0.01 , uncorrected.

\section{Relationship between years of transcription training and brain structure in control regions}

To ensure that our results were specific to the left pars opercularis, we also used partial correlations, controlling for overall cortical volume, to examine the relationship between years of transcription training and brain structure in several control regions: the right hemisphere homolog to the left pars opercularis, and the left and right lateral occipital gyri.

\section{Relationship between brain structure and nonnative phonetic learning in phoneticians}

To determine whether individual differences in skill among the phoneticians predicts aspects of brain structure in this group, we behaviorally tested the phoneticians on their perceptual learning of a synthetic Hindi dental-retroflex contrast. This procedure and the stimuli used were the same as have been used in several studies examining brain structural correlates of phonetic learning (Golestani et al., 2002, 2007), and it has been shown that healthy (nonexpert) individuals who learn to hear this contrast more rapidly have a larger left Heschl's gyrus than do slower learners. We calculated a continuous learning rate measure ( $\mathrm{L}$ scores) on how quickly participants learned to hear the dental-retroflex contrast during an identification-with-feedback training paradigm, and used this to test for relationships between nonnative phonetic learning and brain structure.

\section{Results}

Table 1 provides an overview of the exploratory and hypothesisdriven analyses and results.

\section{Voxel-based morphometry}

VBM showed that phoneticians are more likely than controls to have more white matter in the right Heschl's gyrus $(t=3.34$; $P_{\text {family-wise error corrected }}=0.05$ after small-volume correction) (Fig. 2) comfamily-wise error corrected
pared with nonexperts. There was a similar trend in the left hemisphere, in a region just posterior to left Heschl's gyrus (LHG) $(t=$ 2.80; $p<0.005$ uncorrected) (Fig. 2), at a location where a second or third transverse gyrus would be expected to lie in cases of transverse gyrus splitting/duplication. This more posterior region behind LHG was not included in our a priori regions of interest but is noted here for completeness. There were no gray matter differences between groups.

\section{ROI analyses}

We subsequently examined brain structure in three ROIs: the auditory cortex bilaterally, the left pars opercularis, and the left supramarginal gyrus.

\section{Transverse gyri}

First, to further characterize the VBM finding described above, we macroanatomically defined and labeled (Fig. 1) the left and right transverse gyri of the auditory cortex on anatomically normalized images. Previous findings in healthy, nonexpert adults have shown that people who are faster at learning to hear and identify foreign speech sounds have not only a larger left Heschl's gyrus than slower phonetic learners, but also that they are more likely to have multiple or split left transverse gyri than slower learners (Golestani et al., 2007). Note that the location of the above left hemisphere result (posterior to Heschl's gyrus, at the location of secondary or tertiary transverse gyri in brains where these exist), suggests that phoneticians may be more likely than nonphoneticians to have multiple or split left transverse gyri. Thus, based on previous findings in healthy individuals, and based on the above VBM result, we tested for differences in both transverse gyrus volumes and also for local differences in brain shape, or gross morphology. We found that phoneticians have larger left $\left(t_{(31)}=2.02, p<0.05\right)$ and right $\left(t_{(31)}=3.07, p<\right.$ 0.005 ) transverse gyri compared with nonexperts (Fig. 3 ), but the number of years of phonetic training was not related to the left $(r=-0.33, p=0.19)$ nor right $(r=0.09, p=0.75)$ transverse gyrus volumes within the phoneticians group. The effects we observed were specific to the transverse gyri; additional analyses of the area that Freesurfer labels the "posterior banks of the superior temporal sulcus" (the supratemporal plane behind Heschl's gyrus) found no significant difference for phoneticians compared with controls in volume $\left(t_{(31)}=-1.21, p=0.24\right)$ or surface area $\left(t_{(31)}=-1.65, p=0.11\right)$, and no significant relationship between the number of years of phonetic transcription training and left volume $(r=-0.21, p=0.47)$ or surface area $(r=-0.17, p=$ $0.56)$. Both the whole brain VBM and region of interest analyses therefore focus the effects of interest to the transverse gyri rather than the surrounding area.

We also tested for gross morphology differences in the transverse gyri between groups, and found that phoneticians are more likely to have multiple or split left $\left(t_{(31)}=2.39, p<0.01\right)$ but not right $\left(t_{(31)}=0.19, p>0.05\right)$ transverse gyri than controls (Fig. 4). Specifically, 14 of $17(82 \%)$ of the phoneticians but only 7 of 16 (44\%) of the controls had either a duplicate or a split left transverse gyrus, and 13 of 17 (77\%) of the phoneticians and 11 of 16 $(69 \%)$ of the controls had either a duplicate or a split right transverse gyrus. A formal measure of asymmetry showed the same result: there was rightward asymmetry in the degree of splitting of the transverse gyri in the controls (LI, $0.20 \pm 0.41$ ), whereas in the phoneticians, the degree of splitting in this region was symmetrical (LI, $-0.02 \pm 0.28$ ). There was also a significant group difference in the lateralization index, with phoneticians showing less rightward asymmetry than controls $\left(t_{(31)}=1.77, p<0.05\right.$, onetailed). In support of this finding, a complementary, automated measure of local gyrification (mean cortical folding, or curvature) in the transverse gyri showed a similar result: there was a trend toward lesser right $>$ left asymmetry in the phoneticians compared with the controls $\left(t_{(31)}=1.49, p=0.07\right.$, one-tailed).

There was no relationship between the number of years of phonetic training and degree of left $(r=-0.19, p=0.47)$ nor right $(r=-0.01, p=0.98)$ transverse gyrus splitting within the phoneticians group. 


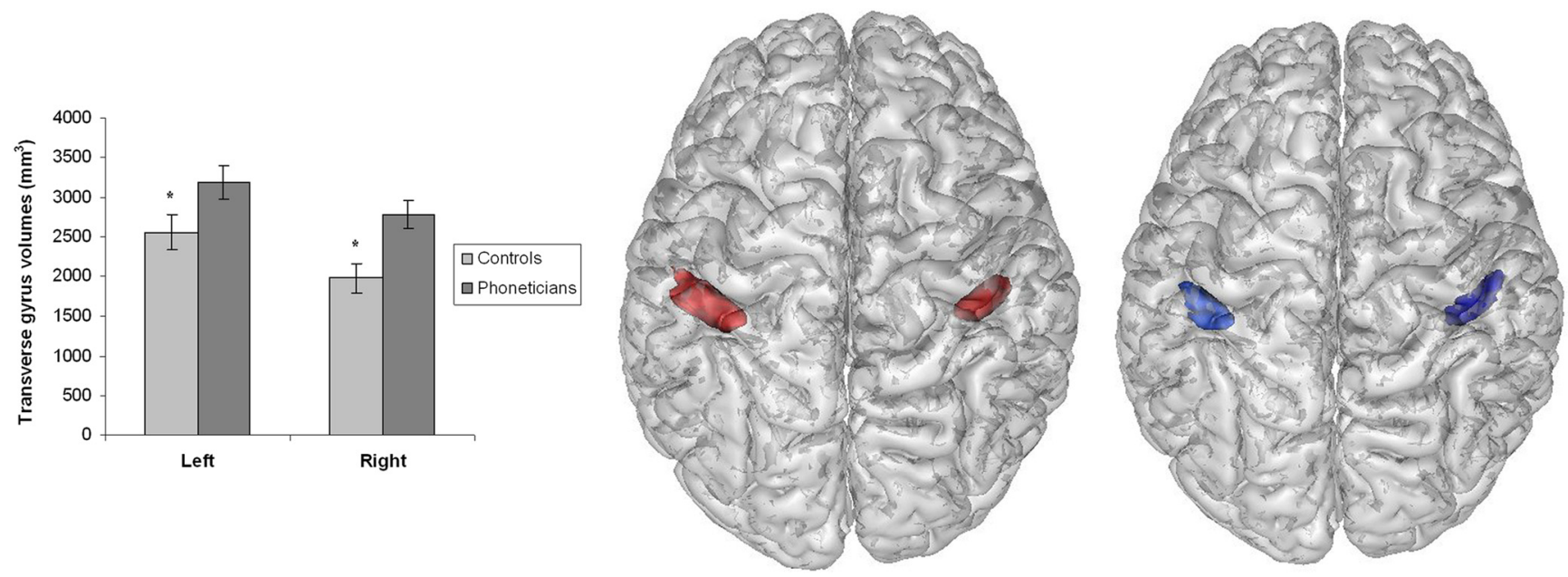

Figure 3. Transverse temporal gyri manual labeling: group differences and fifty-percent thresholded probability maps for phoneticians (red) and nonexperts (blue).

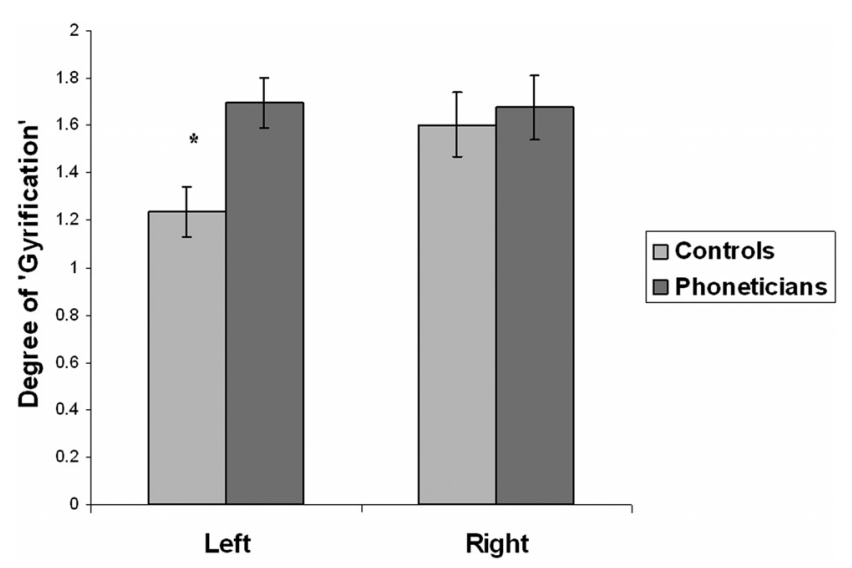

Figure 4. Hemisphere by group means showing degree of transverse gyrus splitting, or duplication, showing greater likelihood of multiple or split left transverse in phoneticians compared with controls.

Left pars opercularis and supramarginal gyrus

Finally, we examined brain structure in two other ROIs known to be involved in phonetic processing: the left pars opercularis and the left supramarginal gyrus. We found that phoneticians had a larger left pars opercularis volume compared with controls $t_{(31)}=2.02$, $p<0.05$, Bonferroni correction). A similar trend was observed for the surface area of the left pars opercularis $\left(t_{(31)}=1.51, p=\right.$ $0.07)$. There were no group differences in the volume or surface area of the left supramarginal gyrus.

Partial correlations controlling for overall cortical volume on the volume and surface area of these regions revealed that within the phoneticians group, years of phonetic transcription training positively predicts the surface area of the left pars opercularis $(r=$ $0.64, p<0.01$, Bonferroni correction) (Fig. 5). A similar trend was observed with the volume of the left pars opercularis $(r=$ $0.49, p=0.04$, Bonferroni correction) (Fig. 5). There were no significant effects of phonetic transcription training on the volume or surface area of the left supramarginal gyrus.

Note that the amount of foreign language experience did not predict various structural measures in the three regions of interest within the phoneticians group (see Other findings, below). Also, partial correlations showed no relationship between transcription training and brain structure in control regions (the right hemisphere homolog of the left pars opercularis, and unre- lated visual control regions, see Other findings, below). Thus, results appear to be specific to the left pars opercularis, and are not driven by more general factors that may be confounded with training.

We also tested the phoneticians on a measure of nonnative speech sound learning to examine whether this measure of phonetic ability may predict aspects of brain structure within the phoneticians group. Specifically, we administered a short training protocol that evaluates the rate of perceptual learning of a synthetic Hindi dental-retroflex contrast (for details of the stimuli and of the training procedure, see Golestani et al., 2002, 2007). Analyses examining relationships between learning rate and brain structure within the phoneticians group did not, however, reveal significant results (see Other findings, below). This may be because this highly preselected expert group had all received extensive training with speech perception and transcription and were all working as professional phoneticians. Consequently, the correlation between brain structure and rate of learning of one particular synthetic nonnative phonetic contrast did not adequately capture existing variability in performance.

\section{Other findings}

\section{Language experience}

The amount of foreign language experience did not predict various structural measures within the phoneticians group. Specifically, partial correlations, controlling for cortical volume, did not show significant relationships within the 15 phoneticians between foreign language experience and the left pars opercularis volume $(r=-0.19, p=0.51)$ or surface area $(r=-0.15, p=$ $0.62)$, and the left supramarginal gyrus volume $(r=-0.43, p=$ $0.12)$ or surface area $(r=-0.30, p=0.30)$. Further, Pearson's correlations did not reveal a relationship across the 17 phoneticians between foreign language experience and left $(r=$ $-0.07, p=0.79)$ nor right $(r=0.03, p=0.91)$ transverse gyrus volumes, and the left $(r=-0.10, p=0.69)$ nor right $(r=0.13, p=0.63)$ transverse gyrus splitting measures (all above tests are two-tailed).

\section{Educational level}

The number of years of education did not predict various structural measures within the phoneticians group. Specifically, partial correlations, controlling for cortical volume, did not show significant relationships within the 15 phoneticians between education and the left pars opercularis volume $(r=-0.34, p=0.24)$ or surface area 

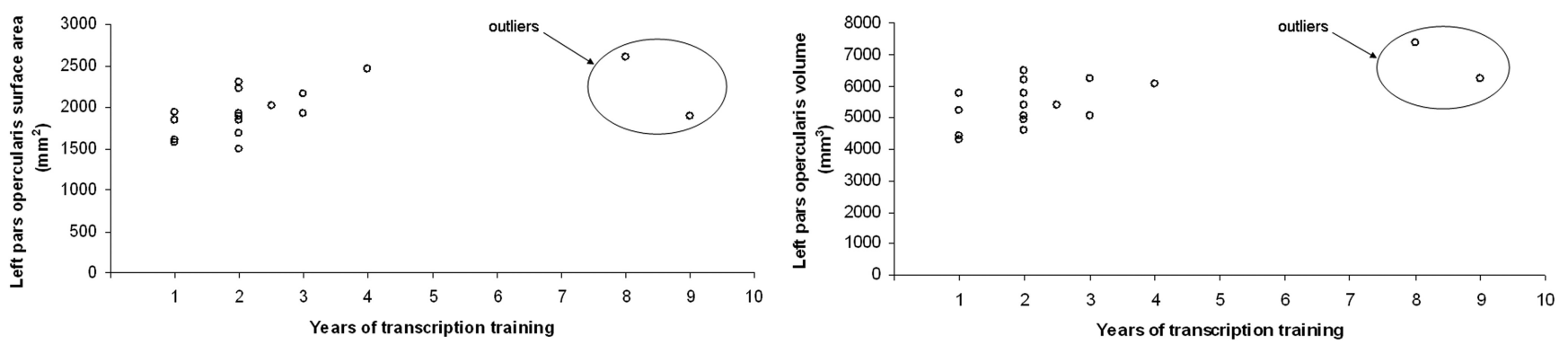

Figure 5. Scatter plots showing relationship between phonetic transcription training and left pars opercularis surface area and volume within the phoneticians group.

$(r=-0.28, p=0.33)$, and the left supramarginal gyrus volume $(r=0.22, p=0.46)$ or surface area $(r=0.07, p=0.82)$. Further, Pearson's correlations did not reveal a relationship across the 17 phoneticians between education and left $(r=0.26, p=0.32)$ or right $(r=0.29, p=0.26)$ transverse gyrus volumes, and the left $(r=-0.09, p=0.74)$ or right $(r=0.21, p=0.41)$ transverse gyrus splitting measures (all above tests are two-tailed).

Relationship between auditory, frontal, and parietal cortex ROI measures and years of transcription training, including two outliers

Results are very similar to those found when excluding the two outliers. Specifically, in all 17 phoneticians, partial correlations controlling for overall cortical volume show no significant relationships between phonetic training and the left supramarginal gyrus volume $(r=-0.10, p=0.72)$ or surface area $(r=-0.01$, $p=0.97)$. As when excluding the two outliers, there is a relationship between transcription training and structure of the left pars opercularis. Specifically, transcription training significantly predicts the volume of the left pars opercularis $(r=0.54, p<0.05$, Bonferroni correction) (surface area was significantly predicted when excluding the outliers), and there is a similar trend for the surface area $(r=0.42, p=0.05)$.

Pearson's correlations also revealed no relationship within the 17 phoneticians between phonetic training and the left $(r=$ $-0.33, p=0.19)$ or right $(r=0.09, p=0.75)$ transverse gyrus volumes, and the left $(r=-0.19, p=0.47)$ or right $(r=-0.01$, $p=0.98)$ transverse gyrus splitting measures.

\section{Relationship between years of transcription training and brain} structure in control regions

There was no relationship between phonetic training and the volume of the right pars opercularis $(r=-0.1, p=0.74)$, the left lateral occipital gyrus $(r=0.02, p=0.96)$, or the right lateral occipital gyrus $(r=0.12, p=0.70)$. Similarly, there was no relationship between phonetic training and the surface area of the right pars opercularis $(r=0.24, p=0.41)$, the left lateral occipital gyrus $(r=0.33, p=0.26)$, or the right lateral occipital gyrus $(r=$ $0.38, p=0.18)$.

\section{Relationship between brain structure and nonnative phonetic} learning in phoneticians

Multiple regression analyses between the $\mathrm{L}$ scores and brain structure throughout the brain in a voxel-based morphometry analysis did not reveal any significant relationship between brain structure and skill within the phoneticians group. Also, there was no relationship within this group between the $\mathrm{L}$ scores and the left $(r=-0.29, p=0.27)$ and right $(r=-0.12, p=0.65)$ transverse gyrus volumes, or with the left $(r=0.02, p=0.96)$ or right $(r=$ $-0.29, p=0.27)$ transverse gyrus splitting measures.

\section{Discussion}

We find complementary evidence for experience-dependent plasticity and for brain structural features that could have existed before the onset of expertise training. The effect of experiencedependent plasticity was observed in the left pars opercularis, which was also found to be larger in phoneticians than matched controls, with the amount of phonetic transcription training within the phoneticians group predicting the surface area of this region. The left pars opercularis lies within the posterior portion of the left inferior frontal gyrus, and together with the more anterior left pars triangularis, it constitutes the classic anterior Broca's speech region. Broca's area is constituted of several anatomically distinct subregions (Amunts et al., 1999; Newman et al., 2003; Gough et al., 2005; Amunts et al., 2010; Kaplan et al., 2010). It has been shown to be involved in a range of linguistic and nonlinguistic tasks, including working memory and sequencing of verbal and nonverbal information (Braver et al., 1997; Gelfand and Bookheimer, 2003), and activation in this region has been shown to be modulated by the complexity of language processing (Sharp et al., 2010). In relation to language, it has been argued that the pars triangularis is involved in semantic processing, whereas the more posterior pars opercularis is involved in phonetic processing (Buckner et al., 1995; Price et al., 1996; Fiez, 1997; Poldrack et al., 1999; Gold and Buckner, 2002; Papoutsi et al., 2009). The finding that transcranial magnetic stimulation of the left pars opercularis disrupts phonetic processing suggests that it is not only implicated but that it is necessary for phonologically based working memory mechanisms (Nixon et al., 2004; Gough et al., 2005). The pars opercularis has also been implicated in subvocal rehearsal in verbal working memory more generally (Paulesu et al., 1993; Démonet et al., 1994), and in the extraction and manipulation of phonetic segments in verbal working memory (Zatorre et al., 1996; Burton et al., 2000). This phonetic segmentation process parallels some of the demands of phonetic transcription, where the ability to effectively parse and identify phonetic segments and to provide a one-to-one correspondence between sounds and symbols is very important. Our findings suggest that the acquisition of expertise performing this very specific linguistic task results in structural plasticity in the associated Broca's area subregion.

We also find gross morphology differences in the left auditory cortex between phoneticians and controls. Previously, we showed that faster phonetic learners have greater left transverse gyrus splitting or duplication than slower learners (Golestani et al., 2007). Here we extend this finding to an expert population and also show that the number of years of phonetic transcription training does not predict this aspect of gross morphology within the phoneticians group. Previous studies have shown transverse 
gyrus morphology to be normatively asymmetric, with greater likelihood of multiple transverse gyri in the right but not the left hemisphere (Pfeifer, 1920; von Economo and Horn, 1930; Beck, 1955; Campain and Minkler, 1976; Chi et al., 1977), as can be seen in our nonexpert, control group $\left(t_{(15)}=1.89\right.$; $p<0.05$; mean right, $1.58 \pm 0.59$; mean left, $1.25 \pm 0.39$ ). Other studies (Penhune et al., 1996; Warrier et al., 2009), however, have shown that the gross morphology of the transverse gyri is quite variable. Our results suggest that this variability may be explained by differences in the populations tested, ones that may in part be explained by systematic differences in domain-specific skills (Leonard et al., 2006). Specifically, we propose that relatively greater gyrification of the left transverse gyrus, which includes primary and secondary auditory cortex in the hemisphere dominant for speech, could be a neuroanatomical marker of skill (whether within normal variability or expertise) in the domain of detailed auditory processing of speech. In line with this interpretation, it has been shown that cortical gyrification at birth predicts functional outcome later in life in preterm infants (Dubois et al., 2008).

As mentioned above, the amount of transcription training did not predict the gross morphology or volume of the left transverse gyrus within the phoneticians group. It is known that the development of the transverse gyrus is established during the 31 st week of gestational age, and that secondary (or tertiary) gyri appear after the 36th week of gestation (Chi et al., 1977). Also, there is no evidence, as of yet, that secondary or tertiary transverse gyri could grow during adulthood, and it has been shown that the development of the transverse gyri is stabilized by the age of seven both morphologically (Leonard et al., 1998; Preis et al., 1999) and myeologenetically (Yakovlev and Lecours, 1967). Our results therefore suggest that the left auditory cortex gross morphology differences could have existed before the onset of phonetic training. Further, it is possible that the presence of such a morphological feature may make it more likely for individuals to become phoneticians or to work in other domains requiring detailed auditory processing. In other words, the presence of such a feature may have an impact on professional specialization and on career choices. Consistent with our interpretation, genetic studies show that Broca's area morphology may be more malleable to experience than Heschl's gyrus morphology. In contrast, Heschl's gyrus shows higher heritability estimates than Broca's area (Peper et al., 2007). In other words, we have found evidence for a potential brain structural intermediate phenotype, or structural marker (Meyer-Lindenberg and Weinberger, 2006), not for disorder or disease, but for a domain-specific aptitude that can, with adequate opportunity and training, lead to expertise.

Greater folding, or gyrification, can arise from developmental mechanisms explained by the radial unit model cortical evolution (Rakic, 1988). According to this model, a higher number of radial units, or minicolumns, results in an increase in cerebral gyrification and surface area. Greater gyrification and surface area, in turn, allow for a greater capacity for establishing new patterns of brain connectivity. This radial unit model could explain individual differences in observed local aspects of brain morphology. Thus, our observations that phoneticians have greater gyrification in the left auditory cortex and overall larger transverse gyri bilaterally could be explained by more complex patterns of brain connectivity in phoneticians compared with controls.

Greater gyrification could also arise from other aspects of microstructural differences between individuals, such as ones that have been proposed to explain left $>$ right asymmetries in core auditory regions. Here, neuronal tract tracing has revealed that there are interhemispheric differences in the organization of the intrinsic microcircuitry of the primary auditory cortex such that more functionally distinct columnar systems are included per surface unit in the left than in the right area 22 (Galuske et al., 2000).

In conclusion, our results demonstrate that extensive training with speech sound analysis, and maybe even with articulationbased approaches, can lead to plastic changes in Broca's area. This has potential implications for training-related remediation for individuals with phonological difficulties (e.g., developmental dyslexia), and in the domain of second language learning. This is in line with other studies showing, for example, that greater musical expertise starting at a young age is associated with more gray matter in the auditory cortex (Gaser and Schlaug, 2003).

\section{References}

Amunts K, Schleicher A, Bürgel U, Mohlberg H, Uylings HB, Zilles K (1999) Broca's region revisited: cytoarchitecture and intersubject variability. J Comp Neurol 412:319-341.

Amunts K, Lenzen M, Friederici AD, Schleicher A, Morosan P, PalomeroGallagher N, Zilles K (2010) Broca's region: novel organizational principles and multiple receptor mapping. PLoS Biol 8:e1000489.

Ashburner J, Friston KJ (2000) Voxel-based morphometry: the methods. Neuroimage 11:805-821.

Beck E (1955) Typologie des gehirns am beispiel des dorsalen menschlichen schlafenappens nebst weiteren beitrage zur frage der links-rechtshirnigkeit. Deutsch Z Nervenheilk 173:267-308.

Braver TS, Cohen JD, Nystrom LE, Jonides J, Smith EE, Noll DC (1997) A parametric study of prefrontal cortex involvement in human working memory. Neuroimage 5:49-62.

Buckner RL, Raichle ME, Petersen SE (1995) Dissociation of human prefrontal cortical areas across different speech production tasks and gender groups. J Neurophysiol 74:2163-2173.

Burton MW, Small SL, Blumstein SE (2000) The role of segmentation in phonological processing: an fMRI investigation. J Cogn Neurosci 12:679-690.

Campain R, Minckler J (1976) A note on the gross configurations of the human auditory cortex. Brain Lang 3:318-323.

Chi JG, Dooling EC, Gilles FH (1977) Gyral development of human brain. Ann Neurol 1:86-93.

Démonet JF, Price C, Wise R, Frackowiak RS (1994) A PET study of cognitive strategies in normal subjects during language tasks: influence of phonetic ambiguity and sequence processing on phoneme monitoring. Brain 117:671-682.

Desikan RS, Ségonne F, Fischl B, Quinn BT, Dickerson BC, Blacker D, Buckner RL, Dale AM, Maguire RP, Hyman BT, Albert MS, Killiany RJ (2006) An automated labeling system for subdividing the human cerebral cortex on MRI scans into gyral based regions of interest. Neuroimage 31:968-980.

Dubois J, Benders M, Borradori-Tolsa C, Cachia A, Lazeyras F, Ha-Vinh Leuchter R, Sizonenko SV, Warfield SK, Mangin JF, Hüppi PS (2008) Primary cortical folding in the human newborn: an early marker of later functional development. Brain 131:2028-2041.

Fiez JA (1997) Phonology, semantics, and the role of the left inferior prefrontal cortex. Hum Brain Mapp 5:79-83.

Fischl B, van der Kouwe A, Destrieux C, Halgren E, Ségonne F, Salat DH, Busa E, Seidman LJ, Goldstein J, Kennedy D, Caviness V, Makris N, Rosen B, Dale AM (2004) Automatically parcellating the human cerebral cortex. Cereb Cortex 14:11-22.

Galuske RA, Schlote W, Bratzke H, Singer W (2000) Interhemispheric asymmetries of the modular structure in human temporal cortex. Science 289:1946-1949.

Gaser C, Schlaug G (2003) Brain structures differ between musicians and non-musicians. J Neurosci 23:9240-9245.

Gelfand JR, Bookheimer SY (2003) Dissociating neural mechanisms of temporal sequencing and processing phonemes. Neuron 38:831-842.

Gold BT, Buckner RL (2002) Common prefrontal regions coactivate with dissociable posterior regions during controlled semantic and phonological tasks. Neuron 35:803-812. 
Golestani N, Pallier C (2007) Anatomical correlates of foreign speech sound production. Cereb Cortex 17:929-934.

Golestani N, Paus T, Zatorre RJ (2002) Anatomical correlates of learning novel speech sounds. Neuron 35:997-1010.

Golestani N, Molko N, Dehaene S, LeBihan D, Pallier C (2007) Brain structure predicts the learning of foreign speech sounds. Cereb Cortex 17:575-582.

Gough PM, Nobre AC, Devlin JT (2005) Dissociating linguistic processes in the left inferior frontal cortex with transcranial magnetic stimulation. J Neurosci 25:8010-8016.

Hartwigsen G, Baumgaertner A, Price CJ, Koehnke M, Ulmer S, Siebner HR (2010) Phonological decisions require both the left and right supramarginal gyri. Proc Natl Acad Sci U S A 107:16494-16499.

Kaplan E, Naeser MA, Martin PI, Ho M, Wang Y, Baker E, Pascual-Leone A (2010) Horizontal portion of arcuate fasciculus fibers track to pars opercularis, not pars triangularis, in right and left hemispheres: a DTI study. Neuroimage 52:436-444.

Leonard CM, Puranik C, Kuldau JM, Lombardino LJ (1998) Normal variation in the frequency and location of human auditory cortex landmarks: Heschl's gyrus: where is it? Cereb Cortex 8:397-406.

Leonard CM, Eckert MA, Kuldau JM (2006) Exploiting human anatomical variability as a link between genome and cognome. Genes Brain Behav 5 [Suppl 1]:64-77.

Maguire EA, Gadian DG, Johnsrude IS, Good CD, Ashburner J, Frackowiak RS, Frith CD (2000) Navigation-related structural change in the hippocampi of taxi drivers. Proc Natl Acad Sci U S A 97:4398-4403.

Meyer-Lindenberg A, Weinberger DR (2006) Intermediate phenotypes and genetic mechanisms of psychiatric disorders. Nat Rev Neurosci 7:818-827.

Milton J, Solodkin A, Hlustík P, Small SL (2007) The mind of expert motor performance is cool and focused. Neuroimage 35:804-813.

Newman SD, Just MA, Keller TA, Roth J, Carpenter PA (2003) Differential effects of syntactic and semantic processing on the subregions of Broca's area. Brain Res Cogn Brain Res 16:297-307.

Nixon P, Lazarova J, Hodinott-Hill I, Gough P, Passingham R (2004) The inferior frontal gyrus and phonological processing: an investigation using rTMS. J Cogn Neurosci 16:289-300.

Papoutsi M, de Zwart JA, Jansma JM, Pickering MJ, Bednar JA, Horwitz B (2009) From phonemes to articulatory codes: an fMRI study of the role of Broca's area in speech production. Cereb Cortex 19:2156-2165.
Paulesu E, Frith CD, Frackowiak RS (1993) The neural correlates of the verbal component of working memory. Nature 362:342-345.

Penhune VB, Zatorre RJ, MacDonald JD, Evans AC (1996) Interhemispheric anatomical differences in human primary auditory cortex: probabilistic mapping and volume measurement from magnetic resonance scans. Cereb Cortex 6:661-672.

Peper JS, Brouwer RM, Boomsma DI, Kahn RS, Hulshoff Poll HE (2007) Genetic influences on human brain structure: A review of brain imaging studies in twins. Hum Brain Mapp 28:464-473.

Pfeifer RA (1920) Myelogenetisch-anatomische Untersuchungen über das kortikale Ende der Hörleitung. Abh Math-Physik Kl sächs Akad Wiss Leipzig 37:1-54.

Poldrack RA, Wagner AD, Prull MW, Desmond JE, Glover GH, Gabrieli JD (1999) Functional specialization for semantic and phonological processing in the left inferior prefrontal cortex. Neuroimage 10:15-35.

Preis S, Jancke L, Schmitz-Hillebrecht J, Steinmetz H (1999) Child age and planum temporale asymmetry. Brain Cogn 40:441-452.

Price CJ, Wise RJ, Warburton EA, Moore CJ, Howard D, Patterson K, Frackowiak RS, Friston KJ (1996) Hearing and saying: the functional neuroanatomy of auditory word processing. Brain 119:919-931.

Rademacher J, Morosan P, Schormann T, Schleicher A, Werner C, Freund HJ, Zilles K (2001) Probabilistic mapping and volume measurement of human primary auditory cortex. Neuroimage 13:669-683.

Rakic P (1988) Specification of cerebral cortical areas. Science 241:170-176.

Sharp DJ, Awad M, Warren JE, Wise RJ, Vigliocco G, Scott SK (2010) The neural response to changing semantic and perceptual complexity during language processing. Hum Brain Mapp 31:365-377.

von Economo C, Horn L (1930) Uber windungsrelief mabe und Rindenarchitektonic der supratemparalflache, ihre individuellen und seitenunterschiede. Neuropsychiatre 30:678-757.

Warrier C, Wong P, Penhune V, Zatorre R, Parrish T, Abrams D, Kraus N (2009) Relating structure to function: Heschl's gyrus and acoustic processing. J Neurosci 29:61-69.

Yakovlev PI, Lecours AR (1967) Regional development of the brain in early life, pp 3-70. Oxford: Blackwell.

Zatorre RJ, Meyer E, Gjedde A, Evans AC (1996) PET studies of phonetic processing of speech: review, replication, and reanalysis. Cereb Cortex 6:21-30. 